\title{
Non-invasive monitoring system to detect sitting people
}

\author{
Daniel Rosato \\ Politecnico di Milano \\ Como, Italy \\ danielalejandro.rosato@mail.polimi.it \\ Sara Comai \\ Politecnico di Milano \\ Como, Italy \\ sara.comai@polimi.it
}

\author{
Andrea Masciadri \\ Politecnico di Milano \\ Como, Italy \\ andrea.masciadri@polimi.it \\ Fabio Salice \\ Politecnico di Milano \\ Como, Italy \\ fabio.salice@polimi.it
}

\begin{abstract}
Determining the position of a body is crucial in the context of the Ambient Assisted Living: knowing if a person is standing, lying down or sitting makes the difference in the process of detecting anomalies due to inactivity. We present a non-invasive monitoring system to recognize if a person is seated using Bluetooth Low Energy technology. The system is composed by devices that can be easily attached to the furniture: a transmitter module that continuously broadcasts Bluetooth signal, and a receiver which collects the RSSi value of the received messages. Finally, a central server evaluates the attenuation of the Bluetooth signal within the $2.4 \mathrm{GHz}$ band, correlating it to the presence of a human body in the proximity of the device.
\end{abstract}

\section{CCS CONCEPTS}

- Applied computing $\rightarrow$ Health care information systems; • Human-centered computing $\rightarrow$ Ubiquitous and mobile computing;

\section{KEYWORDS}

Proximity detection, Non-invasive monitoring system, Bluetooth Low Energy, Ambient Assisted Living

\section{ACM Reference Format:}

Daniel Rosato, Andrea Masciadri, Sara Comai, and Fabio Salice. 2018. Noninvasive monitoring system to detect sitting people. In International Conference on Smart Objects and Technologies for Social Good (Goodtechs '18), November 28-30, 2018, Bologna, Italy. ACM, New York, NY, USA, 4 pages. https://doi.org/10.1145/3284869.3284907

\section{INTRODUCTION}

According to the United Nations report [9], the number of people who aged 60 years old and above is estimated to increase $56 \%$, rising from over 900 million to nearly 2.1 billion by 2050 . This results in an increase in hospitalization requests, that create problems in the

Permission to make digital or hard copies of all or part of this work for personal or classroom use is granted without fee provided that copies are not made or distributed for profit or commercial advantage and that copies bear this notice and the full citation on the first page. Copyrights for components of this work owned by others than ACM must be honored. Abstracting with credit is permitted. To copy otherwise, or republish, to post on servers or to redistribute to lists, requires prior specific permission and/or a fee. Request permissions from permissions@acm.org.

Goodtechs '18, November 28-30, 2018, Bologna, Italy

(C) 2018 Association for Computing Machinery.

ACM ISBN 978-1-4503-6581-9/18/11 ..\$15.00

https://doi.org/10.1145/3284869.3284907 health-care system, forcing a change of the actual paradigm of welfare. This is why more and more older people face old-age at home, while new technologies are increasingly offering solutions to monitor and assist them in order to guarantee a fast intervention in case of home accidents.

From many years a lot of research has been done to detect patterns in the behavior of the people being monitored, and thus, facilitating the recognition of anomalies in their everyday lives [13]. Most of the time, the home anomalies, triggered by both accidents and sudden illnesses, cause an evident change in the usual behavior of the inhabitants, leading to inactivity (e.g. the time spent in the living room is excessively long due to the usual behavior of the monitored person) [7]. Monitoring the daily life of people at home, using non-intrusive environmental sensors, it is possible to infer the Activities of Daily Living that he/she performed [12]. It is therefore necessary to identify the sensors necessary for the detection of anomalies that can be correlated to dangerous situations, and which must therefore trigger an immediate reaction to limit their possible negative consequences. Analyzing the above example, knowing if the monitored person is sitting on the sofa with the TV on or lying on the floor during the long stay in the living room can make the difference to decide if it is necessary to alert somebody. This work present a non-intrusive monitoring system to detect sitting people.

\section{RELATED WORKS}

Technological systems that detect the proximity of a human being have been widely studied in the past [4]. However, none of the solutions available in the literature answer the needs of detecting a human sitting down on a piece of furniture in a non-intrusive way.

Cameras and infrared light have been widely used as human proximity detector. But they can get very expensive and the related sensors are hard to position because the target should always be visible in the camera field of view. They are also sensitive to light, sometimes unable to function properly when it is dark and most importantly may irritate the person being monitored [2].

Capacitive sensors are also used for proximity detection, but they are very noisy, since they are susceptible to changes in the environment like temperature and humidity. Also, they are not very power efficient solutions. Some examples of capacitive sensors used for proximity detection can be found in Karlsson et al. [6] and Togura et al. [11].

On the contrary, Bluetooth technology has not been explored much for proximity detection. This kind of systems usually take 
advantage of a wearable device [8], making it too intrusive for the scope of this paper. However, there have been efforts to use this technology as motion detection [10] and for an indoor positioning system [3] using a wearable free approach, measuring the distortion in the signal caused by a human being present, with positive results.

\section{PROXIMITY DETECTION}

Bluetooth low energy (BLE) is a wireless personal area network technology, used for data transmission among devices. It is intended to be a low power alternative to regular Bluetooth.

BLE enables very low-power battery-operated applications to run for months or even years. They use $50-99 \%$ less power, which means the average beacon can last up to 2 years. This is thanks to the fact that BLE remains in sleep mode constantly except for when a connection is initiated. The actual connection times are only a few milliseconds, unlike Bluetooth which would take 100 milliseconds. The reason the connections are so short, is that the data rates are so high at $1 \mathrm{Mb} / \mathrm{s}$. BLE beacons also have a $60-80 \%$ cheaper cost of operation than competing standards.

The BLE signal is transmitted in the $2.4 \mathrm{GHz}$ radio frequency. This means that the signal may be distorted by interference from specific elements in the environment such as metallic surfaces, glass and water. It is important to note that a human body is about $60 \%$ water, it absorbs signals in the $2.4 \mathrm{GHz}$ band. Taken this into account, by identifying such distortion it is possible to infer human proximity.

With the help of a beacon transmitter that uses BLE technology it is possible to measure the amount of distortion in the signal when a human being is present between the beacon and the receiver. It is possible to use the RSSI (received signal strength indicator) as a measurement value to determine these changes. When a person stands between the devices, the signal is not completely shielded, because Bluetooth signals are omnidirectional. However, the RSSI decreases.

The RSSI can be measured with the Friis transmission equation:

$$
P_{r}=P_{t} *\left(G_{t} * G_{r}\right) * c^{2} /(4 \pi R f)^{2}
$$

This equation gives the power received by an antenna from another antenna that is transmitting a known amount of power at a distance under ideal conditions.

There is a challenge in this approach, as mentioned before, represented by the several environment variables that distort the $2.4 \mathrm{GHz}$ signal of the beacon. Thus, it is important to calibrate the system to identify the right amount of distortion created by the human body.

\subsection{Architecture}

The main goal of the proposed research is to provide a non intrusive solution that can be placed underneath a piece of furniture, considering usability, cost and reliability issues.

BLE beacons meet most of the requirements discussed so far, making it the most suitable technology for the proposed system. Taking the role of beacon and receiver, two Esp32 modules[1] were selected to realize a demo. These devices are small and very versatile, one of them can perform many tasks such as serve as BLE beacon, BLE receiver and also connect to $\mathrm{WiFi}$ and send HTTP requests, which will comprise an important part in the final solution.

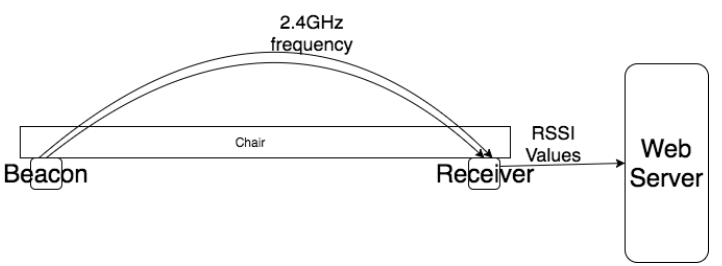

Figure 1: Representation of the Architecture

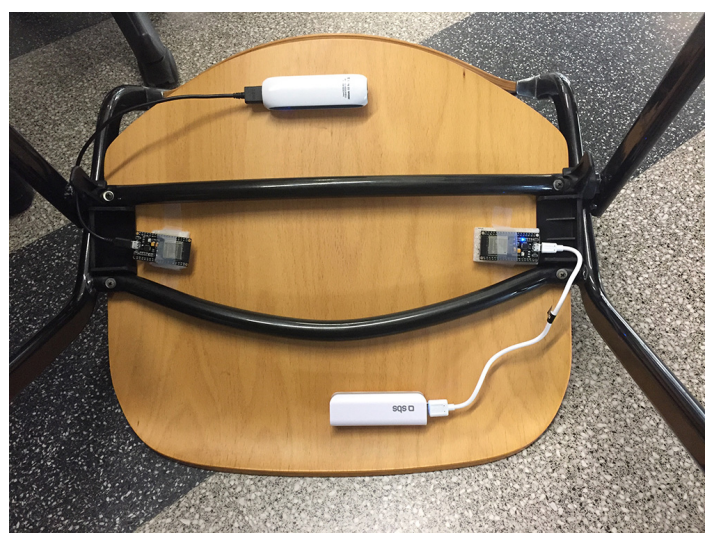

Figure 2: Devices placed underneath a chair

As shown in Figure 1, the proposed solution is composed, in addition to the esp32 modules, of a web server that receives and analyzes in real time the RSSI measurements. The first prototype of the solution uses two power banks to power the microcontrollers, in addition of double sided tape to place them below the furniture (Figure 2).

\subsection{Model Selection}

The problem of detecting a seated person has been modeled as a classification problem. That is, given a set of inputs, a prediction of a qualitative variable (seated or not) is required. Predicting a qualitative response for an observation can be referred to as classifying that observation, since it involves assigning the observation to a category. In this case the input variable is composed by the RSSI measurements taken from the microcontrollers and sent to the web server. The idea is to process these values in real time and assign each observation to one of two categories: seated, not seated.

Two models were chosen to tackle the problem:

- Logistic Regression

- Standard Deviation

It is important to note that both models are supervised training models. This means that a training or calibration phase is needed.

3.2.1 Logistic Regression. To use this approach [5], first it is needed to predict the probability of each category of a qualitative variable as the basis to perform the classification.

This model measures the probability that a response $\mathrm{Y}$ falls into a particular category. The threshold for this decision is previously established (E.g. $P(X)>$ threshold then the prediction is category one, otherwise the prediction is category two). 


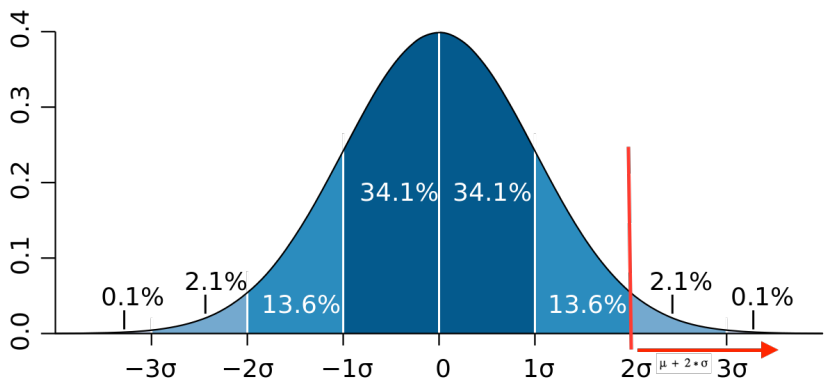

Figure 3: Bell Distribution

The threshold value selected was 0.75 . This is because to avoid false positives as much as possible, a high probability of being seated is required.

To model the relationship between the input variable and the probability of that observation occurring, the logistic function is used. The following function (Equation 2) returns values between 0 and 1.

$$
p(X)=\frac{e^{\beta_{0}+\beta_{1} X}}{1+e^{\beta_{0}+\beta_{1} X}}
$$

To fit the model and estimate the coefficients, a method called Maximum Likelihood is used (Equation 3).

$$
l\left(\beta_{0}, \beta_{1}\right)=\prod_{i: y_{i}=1} p\left(x_{i}\right)+\prod_{i^{\prime}: y_{i^{\prime}}=1}\left(1-p\left(x_{i^{\prime}}\right)\right)
$$

This method tries to find the values of $\beta_{0}$ and $\beta_{1}$ that yields the values that correspond as closely to the individual's observed output. In this case whether the individual is seated or not.

Once the coefficients are estimated, it is a simple matter of computing the probability of being seated or not.

3.2.2 Standard Deviation. Assuming that the data follows a normal distribution. This model consists in finding the standard deviation of the distribution of the sample taken to later find anomalies in new observations.

By calculating the mean and the standard deviation we can identify the values that are not consistent with $95 \%$ of the observations by applying the following formula (Equation 4):

$$
y=\mu+2 * \sigma
$$

Observations that are less than $y$ can be considered anomalies. It is important to note that in this case we only need the upper bound, given that we are only interested in the distortion of the BLE signal and that the RSSI values are going to be measured in $\mathrm{dBm}$ (decibel milliwatts) which will give values lower than zero. This means that we care about anomalies as shown in Figure 3.

\section{CALIBRATION}

As mentioned before, the selected models are supervised training models. This means that they require a set of training data to start predicting and classifying accurately new observations. This phase of training is called calibration and is key for the good performance of the system.

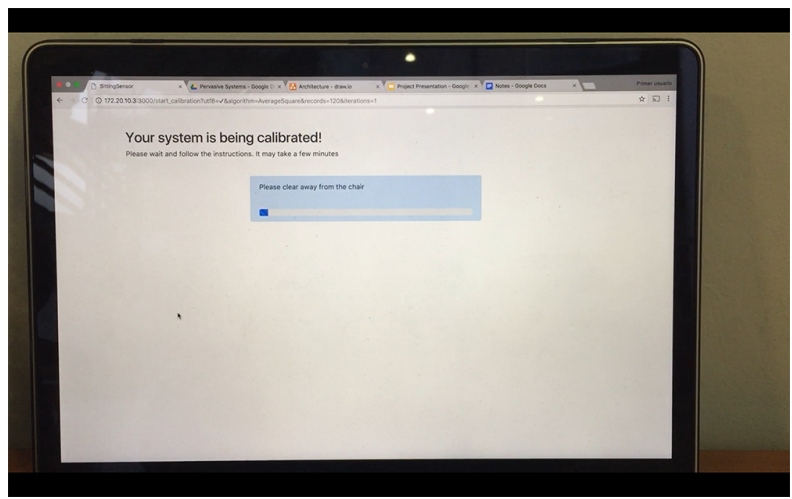

Figure 4: System Being Calibrated

Table 1: Logistic Regression Results - Confusion matrix showing the values collected with Logistic regression as a prediction algorithm.

\begin{tabular}{|l|l|l|}
\hline & Predicted: NO & Predicted: YES \\
\hline Actual: NO & 1342 & 81 \\
\hline Actual: YES & 13 & 540 \\
\hline
\end{tabular}

An important aspect of the solution is that it needs to be easy to use. Taking this into account the system was developed with an auto calibration feature, this means that after it is plugged in underneath the chair, it can calibrate itself and start predicting. The calibration phase can be performed using the web interface of the server (Figure 4); using the same applications it is also possible to register chairs, beacons, and their coupling. Finally, after the calibration phase it is possible to visualize the predictions for every chair in real time.

At this stage the system is collecting enough samples to train the models previously mentioned. Depending on the model selected, different calibration methods can be used (E.g. for the logistic regression model, the system asks the user to sit down and stand up a few times).

\section{EXPERIMENTS}

Several experiments were performed to test both models and the overall performance of the system.

The experiments consisted in testing several archetypes of people when sitting down. Different weights and heights, as well as different genders were analyzed.

In order to collect ground truth data, a member of the team would ask the subjects to stand up or sit down while at the same time signaling the system of the actions of the subject plus his weight, height and gender.

Once enough data was collected and the system trained, then it was used to perform predictions on live observations.

Results for the Logistic Regression approach are shown in Table 1 and Table 2, while the results for the Standard Deviation approach are shown in Table 3 and Table 4: it can be observed that both the models hold an accuracy greater than $90 \%$. 
Table 2: Logistic Regression Results - Performance metrics calculated from Table 1

\begin{tabular}{|l|l|}
\hline Precision & 87.0 \\
\hline Sensitivity & 97.6 \\
\hline Accuracy & 95.2 \\
\hline Specificity & 94.3 \\
\hline
\end{tabular}

Table 3: Standard Deviation Results - Confusion matrix showing the values collected with Standard deviation as a prediction algorithm.

\begin{tabular}{|l|l|l|}
\hline & Predicted: NO & Predicted: YES \\
\hline Actual: NO & 708 & 33 \\
\hline Actual: YES & 27 & 210 \\
\hline
\end{tabular}

Table 4: Standard Deviation Results - Performance metrics calculated from Table 3

\begin{tabular}{|l|l|}
\hline Precision & 86.4 \\
\hline Sensitivity & 88.6 \\
\hline Accuracy & 93.9 \\
\hline Specificity & 95.5 \\
\hline
\end{tabular}

The main difference between them is the Sensitivity, which indicates of all the people that were seated, how much did the system predicted correctly. The logistic regression model has a higher sensitivity than the Standard deviation one.

Although the Precision it is a high value for both the models, over $85 \%$, it is still relatively low compared to the other results. This means that both models suffer from relatively high, in comparison with other values, of false positive rates. That is, from all people labeled as seated, how much they were actually seated.

Both models have a similar Specificity rate. This is the proportion of the true negatives correctly identified.

As mentioned before, both models returned positive results. It is important to mention that while the logistic regression model has a higher accuracy and sensitivity, it also takes longer to calibrate.

\section{DISCUSISON}

The obtained results show that using Bluetooth Low Energy devices such as beacons are a good solution to detect whether a person is sitting or not, even more in the context scenario. However, it is worth consider that all the experiments were performed in friendly settings. The system is very susceptible to noise and therefore to false positives. It relies heavily on the structure of the chair given that there is no standard way of placing the receiver and the beacon, only that they should be close to each other.

Moreover the use of a supervised learning algorithm like logistic regression makes the calibration part tedious and difficult for the user.

These are all opportunities to improve further versions of the system; the next activities will be devoted to add the possibility to consider more beacons for a single chair. This will improve the reliability and the robustness of the system, making it less susceptible to the influence of the environment. Indeed, it is very important to reduce the noise and consequently the false positives, i.e. to improve the precision of the system. The possibility of using a Kalman Filter will be being explored to perform sensor fusion.

\section{CONCLUSION}

This work studies the possibility of using BLE technology for human proximity detection. This system can be really helpful in the framework of Assistive Technologies to retrieve more detailed information about the behavior of people (e.g. how much time a person is spending sitting on a chair) in a non-intrusive way. The low cost and long life of BLE beacons makes them the ideal candidates to be used in a domestic setting. Using the RSSI calculated with the Friis transmission equation, the distortion generated in the signal by a human body positioned between the beacon and the receiver can be identified and sent to a web server.

Machine learning techniques - two different supervised models - were evaluated to analyze the data sent by the receiver, and therefore make a prediction on whether someone is seated or not.

Based on the results obtained by the experiments performed, it can be concluded that the objectives set out in the project were accomplished and the use of BLE technology can be a viable solution for the proximity detection of a human being.

It is important to note that even though the accuracy achieved was high, further work needs to be done to be able to ignore interference by metallic surfaces and other environmental variables.

\section{REFERENCES}

[1] 2018. ESP-32 Module. https://www.espressif.com/en/products/hardware/esp32/ overview. Accessed: 2018-09-01.

[2] Ji Hoon Lee Kwang Yong Shin Yeong Gon Kim Toan Thanh Le Eun Som Jeon, Jong-Suk Choi and Kang Ryoung Park. 2015. Human Detection Based on the Generation of a Background Image by Using a Far-Infrared Light Camera. (2015).

[3] Ramsey Faragher and Robert Harle. 2014. An Analysis of the Accuracy of Bluetooth Low Energy for Indoor Positioning Applications. (2014).

[4] Li Hou, Wanggen Wan, Kang Han, Rizwan Muhammad, and Mingyang Yang. 2016. Human detection and tracking over camera networks: A review. IEEE International Conference on Pervasive Computing and Communications (PerCom) (2016)

[5] Gareth James, Daniela Witten, Trevor Hastie, and Robert Tibshirani. 2013. An Introduction to Statistical Learning with Applications in $R$. Springer.

[6] Nils Karlsson and Jan-Ove Jarrhed. 1993. A Capacitive Sensor for the Detection of Humans in a Robot Cell. IEEE International Conference on Pervasive Computing and Communications (PerCom) (1993).

[7] Andrea Masciadri, Anna A Trofimova, Matteo Matteucci, and Fabio Salice. 2017. Human Behavior Drift Detection in a Smart Home Environment. Stud. Health Technol. Inform 242 (2017), 199-203.

[8] Alessandro Montanari, Sarfraz Nawaz, Cecilia Mascolo, and Kerstin Sailer. 2017. A Study of Bluetooth Low Energy Performance for Human Proximity Detection in the Workplace. IEEE International Conference on Pervasive Computing and Communications (PerCom) (2017).

[9] World Health Organization. 2015. World report on ageing and health. World Health Organization.

[10] Kyohei Sugino, Yusuke Niwa, Shun Shiramatsu, Tadachika Ozono, and Toramatsu Shintani. 2015. Developing a Human Motion Detector using Bluetooth Beacons and its Applications. Information Engineering Express 1, 4 (2015).

[11] Takeshi Togura, Koji Sakiyama, Yasushi Nakamura, and Kazuya Akashi1. 2009. Long-Range Human-Body-Sensing Modules with Capacitive Sensor. (2009).

[12] Fabio Veronese, Andrea Masciadri, Anna A Trofimova, Matteo Matteucci, and Fabio Salice. 2016. Realistic human behaviour simulation for quantitative ambient intelligence studies. Technology and Disability 28, 4 (2016), 159-177.

[13] Fabio Veronese, Hassan Saidinejad, Sara Comai, and Fabio Salice. 2016. Elderly monitoring and AAL for independent living at home: human needs, technological issues, and dependability. In Optimizing Assistive Technologies for Aging Populations. IGI Global, 154-181. 